Mots. Les langages du politique

\title{
Clause sociale : limites et contradictions des réponses syndicales
}

Social Chapter: limits and inconsistencies of trade unions' response

Clásula social : límites y contradicciones de las respuestas sindicales

\section{Sophie Béroud}

\section{OpenEdition}

Journals

Édition électronique

URL : https://journals.openedition.org/mots/7743

DOI : $10.4000 /$ mots. 7743

ISSN : 1960-6001

Éditeur

ENS Éditions

Édition imprimée

Date de publication : 1 mars 2003

Pagination : 23-39

ISBN : 2-84788-027-5

ISSN : 0243-6450

\section{Référence électronique}

Sophie Béroud, «Clause sociale : limites et contradictions des réponses syndicales », Mots. Les

langages du politique [En ligne], 71 | 2003, mis en ligne le 05 mai 2008, consulté le 23 avril 2022. URL http://journals.openedition.org/mots/7743 ; DOl : https://doi.org/10.4000/mots.7743

\section{(c) ENS Éditions}


Sophie BÉROUD ${ }^{\circ}$

\section{Clause sociale : limites et contradictions des réponses syndicales}

La revendication visant à introduire le respect d'un certain nombre de conditions sociales dans les accords commerciaux n'est pas nouvelle dans l'histoire du syndicalisme. En 1881 déjà, la Federation of Organized Trade and Labour Union a tenté de faire pression sur le Congrès pour que celui-ci augmente les tarifs douaniers pratiqués par les États-Unis, afin de réduire les avantages commerciaux dont bénéficiaient $a$ priori les pays où les salaires moyens s'avéraient plus bas ${ }^{1}$. Ces préoccupations, associées à la volonté de pacifier les relations internationales en encourageant la convergence des politiques sociales, ont ensuite été débattues au sein de l'Organisation internationale du travail (OIT), dès sa création en 1919, et de l'ancêtre de l'ONU, la SDN, au cours des années 1920. Pour autant, alors qu'une nouvelle déclaration de l'OIT, à Philadelphie en 1944, rappelait que «le travail n'est pas une marchandise ${ }^{2}$, la référence à des «standards» sociaux se voyait complètement écartée lors du lancement du GATT en 1948.

Le débat a repris et s'est amplifié depuis le début de la décennie 1990, l'Organisation mondiale du commerce (OMC) prenant la suite du General Agreement on Tariffs and Trade (GATT) et affichant clairement sa prétention à traiter l'ensemble des activités humaines comme autant de marchandises devant être soumises à la libre concurrence. Face à une mondialisation agressive pour l'environnement, destructrice d'emplois dans les pays industrialisés et créatrice de précarité, voire de surexploitation des femmes et des enfants dans le Tiers-Monde, les organisations syndi-

- IEP Paris, Celsa-Paris 4.

1. D. Brand, R. Hoffman, 1994, «Le débat sur l'introduction d'une clause sociale dans le système commercial international : quels enjeux ? », Problèmes économiques, 2400, p. 5.

2. L'OIT a été associée à l'ONU, dès la création de celle-ci. Elle est la seule institution tripartite (représentants des salariés, des employeurs et des gouvernements) du système onusien. 
cales sont confrontées au défi d'élaborer des revendications crédibles et de ne pas se laisser enfermer dans une posture de simple rejet. Cet enjeu s'avère d'autant plus criant que l'effondrement des pays dits socialistes a rendu caduque la forte division du syndicalisme international ${ }^{3}$, ouvrant la voie à des mobilisations convergentes, à l'Est comme à l'Ouest, au Sud comme au Nord.

Placées devant l'obligation de renforcer et de renouveler leurs visées stratégiques, des organisations comme la CISL se sont lancées dans la réélaboration de mesures réformistes visant tout à la fois les institutions financières, les accords de commerce et les multinationales. La notion de clause sociale a connu un regain d'intérêt dans ce contexte ${ }^{4}$. Outre la CISL et l'OIT, nombre d'organisations et d'institutions soutiennent désormais la nécessité d'inclure une clause sociale (mais aussi environnementale et démocratique) dans les relations commerciales internationales. ONG, États, associations patronales peuvent ainsi se retrouver apparemment unis sous cette bannière, ce qui se traduit, de façon logique, par une extension du référent pris en compte par chacun. En ce sens, traiter de la clause sociale ne veut pas dire grand chose tant que la lexie n'est pas éclairée par son contexte d'utilisation : la référence peut aussi bien accompagner une volonté de démantèlement des tactiques de dumping social déployées par les entrepreneurs du Sud (les industriels du textile du Nord s'unissant pour mettre fin à «l'avantage injustifié» des bas salaires) que viser, dans les déclarations d'une ONG, la défense des droits les plus élémentaires de la personne humaine.

En dépit de ce flou grandissant lié à un usage pluriel, la revendication d'une clause sociale est devenue un sujet imposé pour les organisations syndicales, dans la mesure où elles entendent démontrer qu'elles sont en

3. Déjà divisé avant la Seconde guerre mondiale, le syndicalisme international s'est fractionné en trois courants à partir de 1949, incarnés par trois confédérations : le courant réformiste, social-démocrate, avec la Confédération internationale des syndicats libres (CISL), le courant communiste avec la Fédération syndicale mondiale (FSM) et le courant chrétien, avec la Confédération mondiale du Travail (CMT). La FSM a été structurellement affaiblie par la disparition de l'URSS tandis que la CMT dispose d'une assise limitée, malgré la présence en son sein de Solidarnosc, de la Confédération des syndicats chrétiens belge, d'organisations latino-américaines et africaines. Les conditions pour une réunification semblent aujourd'hui réunies, bien que les pesanteurs organisationnelles rendent cette nouvelle étape difficile. Voir R. Gumbrell-McCormick, 2000, «Quel internationalisme syndical ? Passé, présent, avenir », Les Temps Modernes, 607, p. 178-206.

4. R. Gumbrell-McCormick attribue l'invention de l'expression exacte de clause sociale (non de l'idée qu'elle recouvre) à Karl Casserini, membre dirigeant de la FIOM dans les années 1950-1960 dans R. Gumbrell-McCormick, 2000, p. 189. 
prise avec les transformations du réel et qu'elles produisent une argumentation informée sur l'ensemble des phénomènes politiques, sociaux et économiques que recouvre le terme de mondialisation. Encore non utilisée dans ses résolutions de congrès par la CGT, la lexie a fait son apparition dans celles de la CFDT en 1995, dans celles de la CFTC et de FO en 1996. Nous avons souhaité questionner, dans cet article, le volet propositionnel présent dans le discours des deux plus grandes confédérations françaises, la CFDT et la CGT, sur la mondialisation. De profondes différences existent, liées à l'évolution récente de chaque organisation, à son positionnement idéologique et à son rapport à l'internationalisme. Afin de replacer ces discours dans leur contexte, nous nous efforcerons d'expliciter, dans un premier temps, ce que recouvre la notion de clause sociale et l'ensemble des mesures dans lequel elle s'insère, de présenter ensuite les critiques qui ont été formulées à son propos avant de détailler l'argumentation mise en avant par la CFDT et par la CGT.

\section{La clause sociale ou la volonté de réguler}

\section{la mondialisation libérale}

La référence à la clause sociale doit être à la fois analysée en elle-même et mise en regard avec un ensemble de propositions complémentaires. Pour une confédération comme la CISL, devenue hégémonique, un objectif essentiel consiste à obtenir dans les traités de commerce internationaux l'obligation pour les parties contractantes de respecter un certain nombre d'exigences sociales en référence aux conventions établies par l'OIT. Lors de la $86^{\mathrm{e}}$ session de la Conférence internationale du travail en 1998, huit conventions ont été rassemblées dans la Déclaration de l'OIT relative aux principes et droits fondamentaux du travail, dans l'objectif de mettre en avant un socle de droits élémentaires, considérés comme universels. La notion de droits, de normes ou de standards sociaux minimaux pourrait être amplement discutée, leur nombre et les dimensions abordées étant relatifs (convient-il d'y inclure le droit à la sécurité et à la santé ? le droit à la formation professionnelle ?) et indissociables du niveau de développement économique. Avec cette déclaration, l'OIT s'est cependant efforcée de définir un bloc indivisible et non négociable de droits démocratiques qui inclut la liberté d'association, la liberté de négociation, l'interdiction du travail des enfants, la condamnation de toute pratique discriminatoire fondée sur la race, le sexe ou la religion ainsi que l'interdiction du travail forcé ${ }^{5}$.

5. Il s'agit des conventions de $1^{\prime}$ OIT n ${ }^{\circ} 29$ et $n^{\circ} 105$ (abolition du travail forcé), $n^{\circ} 87$ 
L'idée défendue ici revient à soutenir que le respect de ces normes élémentaires devrait contribuer à limiter - premier point d'appui - la mise en concurrence généralisée des salariés induite par la mondialisation des échanges et des capitaux. « [...] L'ingérence dans les marché est nécessaire», affirme en ce sens le Guide syndical de la mondialisation publié par la CISL, "pour protéger les droits des populations depuis le village jusqu'au niveau mondial $»^{6}$. Le problème réside, toutefois, dans le fait que l'adoption de normes sociales (dans un cadre législatif) dépend de la seule volonté des gouvernements et que ceux-ci s'avèrent, par la force des choses, beaucoup plus soumis aux conditions imposées par le FMI et la Banque mondiale qu'attentifs aux recommandations de l'OIT. Pire même, les plans d'ajustement structurel prescrits dans les Pays en développement (PED) par ces mêmes institutions internationales, avec la privatisation des services publics, les restrictions budgétaires dans des domaines comme la santé et l'éducation, ont grandement contribué à porter atteinte aux droits de la personne humaine.

Ces difficultés ont conduit la CISL et les fédérations syndicales internationales $(\mathrm{FSI})^{7}$ à réfléchir à d'autres vecteurs d'introduction des normes sociales au cœur du système capitaliste mondialisé, les États n'apparaissant plus, de fait, comme des entités capables d'exercer leur souveraineté. La CISL mène ainsi depuis la fin des années 1990 une campagne pour que l'OMC fasse de la garantie des droits fondamentaux des travailleurs une clause à part entière des accords commerciaux. Parallèlement, la FITBB (Fédération internationale des travailleurs du bâtiment et du bois) s'efforce de convaincre la Banque mondiale d'inclure dans ses accords de prêts (destinés à des projets de développement) une clause contraignante relative au respect des normes minimales du travail. La campagne en faveur des clauses sociales s'adresse donc non seulement aux États, mais aussi aux institutions financières internationales, acteurs centraux dans la globalisation des marchés. Enfin, l'édification d'une ébauche de cadre de négociation au sein des multinationales s'apparente à une tâche hercu-

(sur la liberté syndicale), $\mathrm{n}^{\circ} 98$ (pour le droit d'organisation et de négociation collective), $\mathrm{n}^{\circ} 100$ (pour l'égalité de rémunération entre hommes et femmes), $\mathrm{n}^{\circ} 111$ (contre la discrimination dans l'emploi) et $\mathrm{n}^{\circ} 138$ et $\mathrm{n}^{\circ} 182$ (sur le travail des enfants).

6. CISL, 2001, Guide syndical de la mondialisation, Bruxelles, p. 25.

7. Il s'agit des anciens Secrétariats professionnels indépendants (SPI), liés à la CISL mais formellement indépendants, qui regroupent les fédérations nationales sur la base de la profession ou du secteur d'activité. 
léenne pour le syndicalisme international ${ }^{8}$. La thématique de l'entreprise socialement responsable et des codes de conduite bénéficie à l'heure actuelle d'un effet de mode, avec la promotion du « Contrat mondial» par $l^{\prime} \mathrm{ONU}{ }^{9}$ et la valorisation des accords-cadres conclus dans une douzaine de multinationales par les fédérations syndicales mondiales. Les codes de conduite ne relèvent cependant pas de la négociation collective et demeurent facultatifs ${ }^{10}$. Ils visent à faire admettre aux multinationales que les pratiques de travail mises en œuvre par leurs fournisseurs et par les soustraitants de leurs sous-traitants relèvent directement de leur responsabilité, quelle que soit la législation nationale en vigueur, et jouent sur la recherche de respectabilité dont ne peuvent s'écarter, grâce à la pression d'un certain nombre de mouvements associatifs, les grandes marques commerciales ${ }^{11}$. La possibilité de mener des campagnes internationales contre des enseignes, actions qui reposent sur le boycott des consommateurs ou sur leur préférence pour des produits affichant un label social, est également discutée au sein du syndicalisme international, suite aux démonstrations réalisées par quelques associations américaines de lutte, dont Job with Justice.

8. Ce sont les SPI, avec la FIOM (Fédération internationale des organisations des travailleurs de la métallurgie) et l'ICF (Fédération internationale des travailleurs de la chimie) qui se sont attaqués les premiers, dès les années 1960, au pouvoir croissant des multinationales. Dans l'objectif de coordonner les stratégies syndicales au sein d'une même multinationale, d'établir en fait un «contre-pouvoir multinational», ils ont cherché à instaurer des conseils d'entreprise mondiaux, Voir U. Rehfeld, 2002, «Des Conseils de groupes mondiaux aux comités d'entreprise européens », dans T. Régin, S. Wolikow (dir.), Les Syndicalismes en Europe, À l'épreuve de l'international, Paris, Syllepse, p. 179-183.

9. Avec le «Contrat mondial», proposition émise par Kofi Annam, secrétaire général des Nations unies, au sommet de Davos en janvier 1999, les entreprises s'engagent à faire preuve «d'une bonne citoyenneté mondiale dans tous les endroits où elles ont des activités». Il leur suffit, pour cela, de déclarer par une lettre adressée au secrétaire général des Nations unies qu'elles entendent respecter les normes sociales fondamentales rassemblées dans la déclaration de l'OIT et d'indiquer une fois par an, sur le site du Contrat mondial, quelles mesures concrètes elles ont prises : http:/www.unglobalcompact.org.

10. «Les nouveaux codes sont quelquefois qualifiés d'initiatives privées facultatives (ou volontaires) car ils requièrent un engagement positif de l'entreprise en vue de leur application». Ils s'apparentent, en ce sens, plus à des instruments de relations publiques qu'à de véritables leviers de l'action syndicale : N. Kearney, D.W. Justice, 2000, «Les nouveaux codes de conduite. Quelques questions et réponses pour les syndicalistes» dans CISL, 2001, Guide syndical de la mondialisation, p. 130.

11. N. Klein, 2001, No logo, La tyrannie des marques, Paris, Actes Sud, 574 p. 


\section{Des mesures controversées}

Cet ensemble de propositions illustre l'effort de réactualisation stratégique auquel est impérativement contraint le syndicalisme international dans ses diverses composantes (confédérales et professionnelles). Toutes ne sont pas placées sur le même plan, la CISL, en raison de son ancrage institutionnel, misant avant tout sur la clause sociale et les FSI sur les accordscadres dans les multinationales. Par ailleurs, bien des difficultés ont été pointées, d'ordre pratique et théorique, contribuant à nourrir un scepticisme critique face aux mots d'ordre de l'entreprise responsable ou du commerce éthique. Des voix dénoncent également les limites intrinsèques du lobbying auprès des institutions internationales et les concessions idéologiques que véhicule implicitement l'idée même de réguler l'économie mondialisée.

Des obstacles, tout d'abord, se révèlent de façon manifeste : ébranlée par l'échec de la conférence ministérielle de Seattle en décembre 1999, l'OMC n'a en rien accédé aux pressions de l'OIT pour entreprendre une coopération avec cette dernière et envisager de placer des références aux normes sociales fondamentales du travail dans les accords commerciaux lors de la conférence de Doha, en novembre 2001. La multiplication des accords bilatéraux d'investissement (ABI) restreint d'ailleurs par avance l'impact que pourrait avoir l'introduction d'exigences sociales relativement contraignantes dans les accords multilatéraux. En ce qui concerne les États et plus particulièrement les rapports Nord-Sud, l'application de la clause de la nation la plus favorisée (ou de Système généralisé de préférence par l'UE) jette un voile hypocrite sur des relations commerciales qui, lorsque nécessité marchande fait loi, occultent la question des droits de l'homme et du travail forcé en Chine. Les multinationales, pour leur part, peuvent très bien s'illustrer par la ratification d'un code de conduite non contraignant tout en continuant à réaliser des profits sur les produits fabriqués dans les zones franches d'exportation.

Les objections de fond, quant à ces diverses mesures, portent à la fois sur le bien-fondé du droit d'ingérence et sur les ressorts d'une solidarité internationale concrète. Pour les PED, l'éventuelle adoption de rétorsions commerciales, en raison des conditions de travail pratiquées sur leur territoire, s'apparente à une forme déguisée de protectionnisme, d'autant plus que la comparaison entre les niveaux de productivité n'est pas envisagée ${ }^{12}$.

12. D. Hornan, 2002, «Clause sociale et résistance», Politique, revue européenne de débats, 3, p. 99-107. 
Les vraies responsabilités - celles des multinationales, du FMI, de la Banque mondiale - sont ignorées, tandis que l'opposition entre travailleurs du Nord et du Sud (conséquence des délocalisations) donne lieu à un large traitement médiatique. Ces mesures risquent, de plus, d'aggraver la situation économique des PED, en restreignant le volume de leurs exportations et, par la même, en augmentant le poids de leur dette extérieure. Le pourcentage des salariés qui pourrait bénéficier, en retour, d'une amélioration de leurs conditions de travail, serait vraisemblablement limité dans la mesure où les secteurs se consacrant à l'exportation ne constituent qu'une part restreinte de l'activité dans la très grande majorité de ces pays, la surexploitation sévissant avant tout dans l'économie dite informelle et sur le marché domestique ${ }^{13}$. À quoi rime, de plus, une action syndicale internationale qui n'entraine pas l'assentiment et la participation des acteurs associatifs et syndicaux des entreprises et des pays concernés ? La mention de normes sociales, même couchée sur le papier, ne saurait constituer un levier important si elle n'entre pas en résonance avec des mobilisations locales, régionales et nationales.

Le fait de s'adresser à l'OMC et d'attendre un geste de bonne volonté de cet organisme ${ }^{14}$ soulève, de plus, le problème de la légitimité de cette dernière. Cette remarque se double d'interrogations quant à la possibilité et aux moyens adéquats pour rendre opératoire un contrôle efficace de l'application des normes sociales fondamentales et pour déterminer les mesures de rétorsion.

Enfin, le recours à une labellisation sociale des marchandises (afin d'attester que leur fabrication n'a pas donné lieu à du travail forcé ou à l'asservissement des enfants, par exemple) a pu être envisagé par certaines associations comme un substitut possible à l'introduction problématique d'une clause sociale dans les accords commerciaux internationaux. Mais une telle opération pose une nouvelle fois la question de la définition des critères d'attribution et du contrôle effectif tout au long des filières de sous-traitance ${ }^{15}$. Elle fait dépendre la résolution des inégalités sociales fondamentales, nées dans les rapports sociaux de production, du comportement final des consommateurs occidentaux - en l'occurrence,

13. D. Brand, R. Hoffman, op. cit. p. 10-12.

14. La CISL, la CMT et la CES ont soumis à l'OMC la proposition de constituer un comité consultatif conjoint avec l'OIT pour la mise en œuvre des clauses sociales.

15. M. Capron, 2001, «Labellisation et normalisation sociales», Le Grain de sable, Courriel d'information d'ATTAC, 290. 
plus ou moins limité selon le revenu dont disposent ces derniers -, ce qui revient à attribuer au marché la capacité de s'autocorriger, en dehors de tout cadre conventionnel et de toute législation.

Avant de clore ce rapide tour d'horizon des diverses critiques, il convient d'ajouter qu'au sein même d'un mouvement syndical plus radical que la direction de la CISL des arguments positifs sont avancés. Dans une contribution rédigée pour le premier ouvrage d'Attac, l'ancien secrétaire de l'UITA (Union internationale des travailleurs de l'alimentation), Dan Gallin, avance qu'effectivement il faut mondialiser les droits démocratiques et les droits syndicaux. Outre le fait que ce type de revendication crée «un point de rencontre entre les syndicats et d'autres organisations de la société civile», il justifie son adhésion à «une stratégie de lutte sans faiblesse pour les droits démocratiques » à la lumière de cet argument :

Pour le mouvement ouvrier, les droits démocratiques ne sont pas une question de préférence culturelle : il s'agit d'un intérêt de classe fondamental, car c'est seulement dans la mesure où ces droits sont garantis que les travailleurs peuvent s'organiser pour défendre leurs intérêts et faire avancer un projet de société alternatif ${ }^{16}$.

\section{La CFDT : un usage détaché de toute critique de fond du libéralisme}

Le rappel synthétique des propositions qui entretiennent un lien étroit avec la notion de clause sociale et des débats qui les concernent nous permettent d'aborder, de façon relative, le discours produit sur ce thème par les deux plus grandes confédérations syndicales françaises. Dès son $43^{\mathrm{e}}$ Congrès de 1995 à Montpellier et plus encore lors de son $44^{\mathrm{e}}$ Congrès à Lille, en 1998, la CFDT a souscrit à l'objectif de «parvenir à une mondialisation maitrisée par des régulations $»{ }^{17}$ en renforçant la coopération entre l'OIT et l'OMC, et par là même «l'intégration de la clause sociale et environnementale dans les politiques de libéralisation des échanges et des investissements».

16. D. Gallin, 1999, «Réinventer la politique du mouvement syndical», dans ATTAC, Contre la dictature des marchés, Paris, Syllepse, p. 107.

17. CFDT, 1998, «Le syndicalisme face au défi de la mondialisation», projet de résolution, Syndicalisme Hebdo, 2709, p. 14. Idem pour les citations suivantes. 
Un paragraphe de la résolution votée par le congrès expose :

La CFDT s'inscrit pleinement dans la campagne de la CISL en faveur d'une clause sociale non protectionniste dans les accords commerciaux et sur les investissements, pour assurer le respect des droits fondamentaux de l'homme au travail définis par les conventions internationales de l'OIT et permettre à l'ensemble des travailleurs de profiter équitablement de la mondialisation des échanges.

Les réticences émises par les gouvernements et les organisations syndicales des PED sont ici écartées par l'invocation rassurante d'une «clause sociale non protectionniste» tandis que la suite de la résolution affirme la nécessité de travailler «en alliance avec les ONG de développement et les associations de consommateurs». Le ton n'est donc pas à la critique, mais plutôt à l'affirmation de la place prise par la confédération dans ce type de démarche. Au $45^{\mathrm{e}}$ Congrès de l'organisation à Nantes en mai 2002 et contrairement à l'intérêt porté à cette dimension lors des précédentes assises de Lille, l'analyse de la mondialisation n'a pas fait l'objet d'une résolution votée, mais d'une simple déclaration non amendable tandis qu'une séance a été consacrée à la projection d'un film sur les entreprises «socialement responsables » et à la présentation de quelques accords-cadres (dont l'accord UITA/BSN). Comme à Lille, la nécessité de sortir du «débat dépressif» sur la mondialisation a été affirmée, afin de «ne pas se cantonner dans l'énoncé des refus». Selon la direction de la CFDT, la mondialisation porte en elle des promesses de croissance et d'émancipation. Malheureusement, elle comporte aussi des «risques» et des «imperfections». Face à ces dernières, la «société civile» et les «forces sociales» ont un rôle à jouer, afin de promouvoir une stratégie de développement durable.

Dans cette optique, la CFDT n'écarte aucun moyen d'action. Dans le paragraphe de la déclaration qui est consacré au thème des droits sociaux, tout un panel de mesures est avancé. Il s'agit, en premier lieu, d'accroitre les pouvoirs de l'OIT pour que celle-ci «soit en mesure de faire respecter les conventions qui en fondent la légitimité». En second lieu, le syndicalisme doit «conquérir et développer de nouveaux espaces d'intervention», ce qui passe, face aux multinationales, par la promotion des accords-cadres, des labels sociaux et... des rapports de forces. En troisième lieu, la CFDT envisage de peser sur les acteurs financiers «pour soutenir l'investissement socialement responsable, notamment à partir de l'épargne des salariés». Enfin, une dernière orientation concerne les modes d'intervention propres aux consommateurs, avec l'engagement de 
la centrale dans le collectif «De l'éthique sur l'étiquette», ce qui revient à promouvoir la pratique des labels sociaux ${ }^{18}$.

Une des originalités du discours de la CFDT sur ce thème consiste à considérer que tous les moyens (accord-cadre, clause sociale, label social, fond d'épargne salariale) servent, sans qu'il y ait besoin ni de les hiérarchiser ni de les distinguer sur la base de leur fonction et de leurs implications, le même objectif d'amélioration des droits des travailleurs de par le monde. Le doute quant à l'efficacité, à la praticabilité, aux résultats obtenus, n'est pas de mise. Surtout, la défense des fonds d'épargne socialement responsable est introduite dans cette liste, comme s'il relevait de la simple évidence qu'un instrument financier constitue un levier de la lutte syndicale au même titre que, par exemple, l'instauration d'un cadre conventionnel dans l'entreprise ${ }^{19}$. Dans une brochure éditée à l'occasion $\mathrm{du} 45^{\mathrm{e}}$ Congrès de Nantes, Agir sur la mondialisation ${ }^{20}$, trois terrains d'actions font l'objet d'une présentation plus détaillée : les institutions financières, les entreprises et multinationales, les relations interétatiques. L'idée d'introduire plus de régulation dans l'économie mondialisée traverse toute la brochure dont le style reste très descriptif, essentiellement informatif. Quatre objectifs sont pointés dès l'introduction : assurer, pour tous, un travail décent, l'accès aux biens publics mondiaux, améliorer la gouvernance mondiale, promouvoir le développement durable. Le choix de ces termes est illustratif de la volonté de la direction de la CFDT de s'approprier le vocabulaire en usage dans les institutions internationales, en particulier à l'OIT et à l'ONU. Le chapitre sur les institutions internationales et, de façon parallèle, les espaces du syndicalisme international donne lieu, une nouvelle fois, à une présentation sans accroc. Ainsi, le TUAC (la commission syndicale consultative au sein de l'OCDE) est loué pour la «réelle valeur ajoutée» qu'il apporte «dans l'élaboration des positions syndicales internationales, notamment sur les questions macro-économiques ». Il est particulièrement significatif que le soutien apporté par le TUAC à l'AMI (Accord sur les investissements multilatéraux) ne soit pas mentionné ici. Dans la sphère internationale, il n'existe donc pas de

18. CFDT, juin 2002, "Déclaration "Le monde que nous voulons"», Syndicalisme Hebdo, 2892, p. 88-93.

19. Cette proposition est avancée de longue date par la CFDT comme en a attesté le colloque sur «Les transformations du capitalisme et les leviers de l'action syndicale» organisé au Conseil national de la CFDT en octobre 1999.

20. CFDT, mai 2002, Agir sur la mondialisation, Paris, CFDT Productions, 78 p. 
grave problème, sinon des régulations à renforcer sur la base de l'existant $^{21}$.

Le discours sur les normes sociales s'est légèrement modifié, même si des constantes demeurent comme l'hypothèse qu'une évolution de l'OMC susceptible d'assurer le «rééquilibre des échanges» relève du probable :

Tout en soutenant l'action de la CISL, la CFDT reste très prudente sur la question des sanctions. S'il faut entendre les craintes des pays du Sud, il ne faut pas renoncer à la promotion des droits sociaux fondamentaux. Mais cette promotion peut se conduire par des actions incitatives mieux que par des sanctions ${ }^{22}$.

Dans une autre brochure éditée au même moment par la CFDT sur le développement durable ${ }^{23}$, le respect des droits fondamentaux, via l'inclusion de clauses sociales et environnementales dans les échanges commerciaux, est présenté de façon plus ferme :

Il s'agit que dans chaque pays, et en particulier les pays en voie de développement, l'ensemble des travailleurs puisse profiter de façon équitable des fruits de la mondialisation des échanges ${ }^{24}$.

La notion d'équité vient compléter ici l'usage des lexies régulation, responsabilité sociale, engagement éthique. L'heure n'est plus à la mise à jour des contradictions entre le capital et le travail, mais bien à l'organisation d'une pression syndicale, en coopération avec les ONG, pour «introduire la notion d'éthique dans le management des entreprises», « souligner que les entreprises ne doivent pas avoir seulement le souci du profit mais aussi celui du cadre social et environnemental dans lequel elles évoluent» ${ }^{25}$ : codes de conduite, accords-cadres, consommation éthique sont célébrés comme autant de vecteurs pertinents, sans que les débats relatifs à ces instruments ne soient présentés de façon raisonnée.

21. Parmi nombre de commentaires édifiants sur le fonctionnement des institutions internationales, on peut lire celui-ci : « Les sommets du G8 constituent un outil important de gouvernance mondiale en matière économique», ou encore au sujet du FMI et de la Banque mondiale : « Or ces programmes [d'ajustement structurel], draconiens sur le niveau des dépenses publiques, ont été critiqués pour avoir retardé l'essor des services d'intérêt général [santé, éducation] ». On mesurera, au travers de ces euphémismes, toute la distance qui sépare la CFDT des mobilisations altermondialistes, dans CFDT, mai 2002, Agir sur la mondialisation, p. 17 et p. 27.

22. CFDT, mai 2002, Agir sur la mondialisation, p. 25.

23. CFDT, mai 2002, Le Développement durable, l'avenir de la planète, Paris, CFDT Productions, $139 \mathrm{p}$.

24. Ibid., p. 53.

25. CFDT, mai 2002, Agir sur la mondialisation, p. 31. 
De ce point de vue, la priorité de la CFDT semble passer par la démonstration qu'elle agit en phase avec les propositions émises au niveau international, ce qui se traduit par une reprise a-critique du vocabulaire produit par des institutions telles que l'OIT et surtout telles que l'OMC, le FMI et la Banque mondiale.

\section{La CGT : insérer la clause sociale dans un ensemble de changements structurels}

Le discours de la CGT sur le thème de la clause sociale se distingue de façon assez nette de celui de la CFDT, bien que la remise en cause de ses anciennes solidarités internationales (avec le départ de la FSM entériné en décembre 1995) et son entrée dans la CES aient bouleversé les principales références de la centrale dirigée par Bernard Thibault. Le document d'orientation (faisant office de résolution) voté par les congressistes lors des 46 ${ }^{\mathrm{e}}$ Assises de Strasbourg en février 1999 affirme une adhésion de principe à la norme sociale (employée ici en substitution à clause sociale):

Ayons de nouvelles exigences, imposons de nouvelles normes internationales, européennes, nationales dans la production de l'échange. Exigeons que la «norme sociale» s'impose car «le travail n'est pas une marchandise» comme les États l'ont déclaré, lors du Congrès de Philadelphie refondant l'OIT après la guerre. Exigeons le respect par tous les États de toutes les conventions fondamentales de l'OIT. Le travail doit être organisé sur la base d'un contrat spécifique, d'une maitrise collective des conditions de l'activité créatrice de l'homme.

Le ton se veut nettement volontariste et la construction des phrases semble consacrer les syndicats comme les acteurs principaux de cette démarche. Le document rappelle à ce propos que ce sont «les artistes, les opinions publiques et le mouvement social» qui ont mis en échec l'AMI secrètement négocié à l'OCDE. Une telle vision s'avère également confirmée par la formulation de commentaires acerbes sur le fonctionnement des organismes internationaux, lesquels doivent être «réformés en profondeur».

Les organisations syndicales comme d'autres forces sociales s'insurgent face aux politiques néo-libérales favorables aux sociétés transnationales imposées par la Banque mondiale, le FMI, l'OMC et l'OCDE. 
En ne dissociant pas son approche des normes sociales d'une critique générale des institutions financières internationales, le document du $46^{\mathrm{e}}$ Congrès propose une analyse plus complexe de ce que pourrait être l'édification d'un droit public international. Il convient, d'une part, de dépasser la simple formulation de «vœux», de «déclarations de principes», de «codes de bonne conduite». Gagner de nouveaux droits pour les travailleurs du monde entier, et en particulier des PVD, implique d'obtenir «des mécanismes de recours, d'application et de contrôle, à l'échelle internationale», avec, pour commencer, la reconnaissance du droit de négociation dans toutes les entreprises, les branches et les pays. L'évocation des normes sociales internationales recouvre, dès lors, un référent plus large que celui de clause sociale (dans le sens retenu par l'OIT et la CISL), avec l'association des droits fondamentaux aux droits sociaux et aux normes sociales de développement. De plus, il est clairement exposé que la définition de normes sociales internationales ne peut être détachée d'une réflexion relative à son articulation avec les règles sociales nationales - le principe de la clause la plus favorable aux salariés étant ici défendu - et qu'elle est à situer dans un projet plus large de «réorientation de l'organisation des marchés». "Sans co-développement, sans nouveau partage des ressources», énonce un des paragraphes du document, «toute norme sociale serait illusoire».

Dans une brochure trilingue, postérieure au $46^{\mathrm{e}}$ Congrès et intitulée Planète plein emploi, réforme des institutions financières internationales $^{26}$, «l'apport du syndicalisme dans le débat sur la régulation des relations internationales » donne lieu à un examen plus fouillé. L'introduction reprend le slogan du mouvement altermondialiste depuis Seattle, «le monde n'est pas une marchandise» et se situe dans une optique clairement offensive : « Nous avons ouvert des brèches dans cet ensemble économique et idéologique que l'on nomme libéralisme». Le processus de mondialisation correspond certes à une «extraordinaire évolution des forces productives», mais il n'a en rien mis fin au rapport dominants / dominés, aux «intérêts toujours contradictoires entre le travail et le capital». Cette qualification ouverte du contexte international dans lequel doit se développer l'action syndicale permet d'énoncer clairement les finalités poursuivies. En ce sens, il ne s'agit pas ici de réguler la mondialisation (bien que la notion de régulation soit reprise en d'autres endroits de la brochure) mais, en solidarité avec les

26. CGT, décembre 2000, Planète plein emploi, Réforme des institutions financières internationales, Montreuil, Espace international de la CGT, 75 p. 
peuples en révolte contre les nouvelles formes d'exploitation, instituées au nom du libéralisme $[\ldots]$ de contribuer au renforcement d'un mouvement social universel capable non seulement de bousculer l'ordre des choses mais de faire naitre une alternative ${ }^{27}$.

Ce mouvement social, divers, composite, doit sortir d'une dépendance vis-à-vis des gouvernements, pour s'atteler à la «construction de nouvelles règles internationales» et obtenir « le plein emploi solidaire partout dans le monde». Plusieurs propositions sont énoncées en conséquence. Nous ne retiendrons que deux axes, en relation au thème de la clause sociale, laissant de côté des revendications relatives à l'annulation de la dette du Tiers-Monde, à la taxation des mouvements de capitaux et aux biens publics internationaux ${ }^{28}$. En premier lieu, si la suppression, jugée stérile, des institutions financières internationales (BM, FMI, OMC) se voit écartée, leur indispensable démocratisation alimente de longs passages de la brochure. Cette démocratisation signifierait une véritable implication des PED en leur sein (ce qui est une façon de commencer à aborder le problème du protectionnisme lié à la clause sociale). Elle ne saurait être confondue avec les faux-semblants des initiatives actuelles de l'ONU en direction des ONG et des entreprises, lesquelles présentent un réel danger de privatisation rampante, sous couvert d'une plus grande ouverture à la «société civile».

En second lieu, la défense de la clause sociale proprement dite n'est pas séparable de la définition de mesures d'accompagnement. La brochure de la CGT rappelle comment les firmes multinationales se servent des délocalisations pour mettre en concurrence les salariés des différents pays. Face à ces méthodes, les conventions de l'OIT, non ratifiées par toutes les grandes puissances du Nord d'ailleurs, demeurent de peu d'utilité concrète. Elles risquent de se transformer en outils cyniques si des pays comme les États-Unis se servent du prétexte du non-respect des normes sociales pour modifier leurs tarifs douaniers ou réduire encore plus leurs aides publiques au développement. Le danger de protection-

27. Ibid., p.3.

28. Il est intéressant de noter que la CGT adopte la lexie biens publics internationaux (ou mondiaux ou biens communs mondiaux), lancée par le Programme des Nations Unies pour le développement (PNUD), ce qui la rapproche sur ce point de la CFDT. Pour autant, cette dernière semble vouloir prévenir la charge potentiellement subversive de la lexie. Dans la brochure Agir sur la mondialisation, «[...] les dérives consistant à vouloir englober dans cette notion "porteuse" toutes les causes à défendre» se voient décriées (dans CFDT, mai 2002, p. 9). 
nisme existe bel et bien et pour convaincre les organisations sociales des PED du bien-fondé de ces normes, il s'avère indispensable de réfléchir à des mesures d'accompagnement (pour subvenir aux besoins élémentaires des familles où les enfants travaillent, par exemple) et de «créer les conditions du développement de ces pays $\gg{ }^{29}$.

Le discours de la CGT sur la thématique de la clause sociale s'avère à la fois plus ciblé et plus distancié que celui de la CFDT, vis-à-vis du discours produit par les institutions internationales et même par la CISL. La brochure précitée n'aborde pas la question de la responsabilité éthique de l'entreprise ni des labels sociaux, mais elle permet de saisir le cadre d'analyse qui permettrait d'y répondre. Dans un débat organisé par la revue Ressources humaines et management, Maryse Dumas, secrétaire de la CGT, met en doute le véritable contenu de la notion de «responsabilité sociale» :

Il y a fort à parier, avance-t-elle, que l'éthique sociale ne se rabougrisse en un argument de marketing supplémentaire, soit à des fins publicitaires, soit pour drainer des fonds supplémentaires, on pense ici évidemment aux fonds de pension et à l'épargne salariale ${ }^{30}$.

Les dangers de «récupération» et d'instrumentalisation des bons sentiments sont ici clairement dénoncés. Plus que sur la politique européenne où son intégration dans la CES lui impose de souscrire à la parole officielle de l'organisation, la CGT continue à appuyer son analyse des rapports sociaux au niveau international sur une grille de lecture qui est la sienne depuis longtemps, fondée sur la prise en compte de l'antagonisme capital / travail. Le repositionnement stratégique de l'action syndicale face au processus de mondialisation semble ainsi passer, et de façon prioritaire, non par la révision des outils d'analyse mais par la construction de nouvelles mobilisations, dimension jugée indispensable pour faire de toute proposition de norme ou de clause sociale un mot d'ordre efficace.

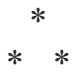

29. CGT, décembre 2000, Planète plein emploi, Réforme des institutions financières internationales, p. 22.

30. M. Dumas, avril 2002, «L'entreprise au défi de l'éthique sociale», Le Peuple, 1558, p. 11. 
La comparaison entre la façon dont la CFDT et la CGT abordent la discussion sur la clause sociale montre à quel point celle-ci sert de miroir à une analyse plus large du processus de mondialisation et à une réflexion stratégique sur les potentialités d'un nouvel internationalisme. Le débat dans les organisations syndicales françaises connait un retard certain par rapport aux échanges qui se déroulent dans les rencontres du mouvement altermondialiste comme le Forum social de Porto Alegre. La dépendance à l'égard d'un vocabulaire forgé en d'autres lieux, imposé le plus souvent par les institutions internationales, s'avère également flagrante. Pourtant, même encore à l'état d'ébauche, les réflexions entourant la clause sociale s'avèrent révélatrices d'orientations divergentes. La CFDT reprend l'ensemble des lexies et des thématiques caractéristiques d'un projet d'aménagement du capitalisme mondialisé dont les mécanismes principaux ne sont pas contestés. L'homologie de ses propositions avec celles de la CISL et de l'OIT lui sert à illustrer sa propre ligne, celle d'une confédération ayant délibérément choisi d'accepter les politiques libérales, tout en cherchant à les amender. La CGT manie avec précaution, quant à elle, les nouvelles lexies, et étaye son discours en recourant à des concepts puisés dans un répertoire plus classique, lié à sa propre histoire. Elle est cependant placée, tout comme la CFDT, devant l'obligation de forger de nouvelles propositions susceptibles d'avoir prise sur l'évolution du réel. 